\title{
哥iresalce
}

\section{O uso de tecnologias virtuais no cuidado à criança e ao adolescente com câncer: Scoping Review}

\author{
The use of virtual technologies in the care of children and adolescents with cancer: scoping \\ review
}

\section{Guilherme Henrique da Silva ${ }^{1}$, Heloisa Cristina Figueiredo Frizzo²}

\begin{abstract}
Resumo
Objetivo: Identificar evidências sobre o uso de tecnologias virtuais como estratégia terapêutica na otimização do cuidado à criança e ao adolescente com câncer. Método: Trata-se de uma investigação Scoping Review realizada nas bases de dados Bireme, Portal.periódicos.CAPES, PubMed e SciELO, identificando as literaturas existentes conforme as etapas do método e os critérios de inclusão e exclusão, sendo incluídos na revisão de escopo sete artigos. Resultados: Realizado mapeamento dos dados dos sete artigos e os resultados foram sintetizados a partir do identitário Tecnologias da Informação, sistematizando em categorias: I) Sistema de informação em saúde; II) Desenvolvimento de recurso virtual; e III) Processamento e análise digital de imagens. Conclusão: As tecnologias virtuais estão interconectadas nas perspectivas de tratamento à criança e ao adolescente com câncer, condizentes às tecnologias de informação, proporcionando assistência, cuidado e qualidade de vida, expressando-se através de ferramentas e organização no processamento de informação, exploração de novos métodos e desenvolvimento de recursos digitais.
\end{abstract}

Palavras-Chave: Câncer; Assistência Integral à Saúde; e-Saúde

\begin{abstract}
Objective: To identify evidence on the use of virtual technologies as a therapeutic strategy in the optimization of care for children and adolescents with cancer. Method: This is a Scoping Review research carried out in the databases Bireme, Portal.periódicos.CAPES, PubMed and SciELO, identifying the existing literature according to the steps of the method and the inclusion and exclusion criteria, being included in the scoping review seven articles. Results: Carried out mapping of the data of the seven articles and the results were synthesized from the identity of Information Technologies, systematizing into categories: I) Health Information System; II) Virtual resource development; and III) Digital image processing and analysis. Conclusion: The virtual technologies are interconnected in the perspectives of treatment to the child and the adolescent with cancer, compatible with information technologies, providing assistance, care and quality of life, expressing themselves through tools and organization in information processing, exploration of new methods and digital resource development.
\end{abstract}

\footnotetext{
1 Graduando em Terapia Ocupacional. Universidade Federal do Triângulo Mineiro (UFTM). Email: guisivato@gmail.com 2 Doutora. Universidade Federal do Triângulo Mineiro (UFTM). Email: heloisa.frizzo@yahoo.com.br

Correspondência: Dept. Terapia Ocupacional UFTM - Rua Vigário Carlos, 100, Prédio do Centro de Pesquisas Prof. Aluízio Rosa Prata, 4ำ piso, Sala 439. Abadia. Uberaba, MG - Brasil. CEP: 38025-180
} 
Keywords: Cancer, Comprehensive Health Care, e-Health.

\section{Introdução}

As tecnologias constantemente incorporam-se às ações de saúde e educação, efetivando conhecimentos e incentivos às práticas saudáveis $\mathrm{e}$ otimizadoras, advindos de habilidades de cuidado e manutenção da saúde dos sujeitos ${ }^{1}$.

Dentre os recursos tecnológicos no contexto virtual na área da saúde, destacase sua inserção nos cuidados oncológicos pediátricos em busca da promoção da informação, comunicação, otimização do tratamento e apropriação no cotidiano da criança e do adolescente.

Compreendendo os planos de cuidado à criança e ao adolescente com câncer, salienta-se a atuação da equipe multiprofissional juntamente com 0 envolvimento da família, tornando recíproca a rede de suporte e adesão aos processos de hospitalização. O diagnóstico e o tratamento do câncer na vida destes sujeitos provocam rupturas nos seus cotidianos, principalmente frente ao desconhecido, vivenciando e processando experiências drásticas².

O processo de adoecimento pode ser temporário ou permanente de acordo com o quadro clínico e o curso da doença. A morbidade interfere na vida e nas ocupações da pessoa que adoece em suas formas, funções e significados.
Quando ocorrem na infância e adolescência, as doenças tendem a restringir o brincar, o lazer, a educação, a sua participação social e o planejamento do futuro ${ }^{3}$.

Analisando a totalidade atual dos casos oncológicos no Brasil, o Instituto Nacional de Câncer (INCA), aponta que a incidência do câncer infantojuvenil corresponde à mediana de $3 \%$ na idade de 0 a 19 anos, que representa 139,99 casos por milhão. Dentre os principais diagnósticos observados no Brasil, destacam-se as seguintes doenças: leucemia $(25,6 \%)$, neoplasias malignas epiteliais $(14,1 \%)$, linfomas $(13,6 \%)$ e tumores do sistema nervoso central $(13,3 \%)^{4}$.

A detecção precoce do câncer pode viabilizar as chances do diagnóstico, tratamento e cura, minimizando a morbidade. Para isto, é necessário redes de serviços de saúde e adiantamento do planejamento terapêutico oncológico com base no quadro clínico e estadiamento do câncer, analisando sempre as condições de saúde do paciente. O tratamento pode ser associado à quimioterapia, radioterapia e cirurgia e, também, pela abordagem multidisciplinar, integralizando os cuidados de maneira efetiva, como a promoção do tratamento, reabilitação e cuidados paliativos $^{5}$. 
A empregabilidade das Tecnologias de Informação e Comunicação (TICs) na saúde possibilita o envolvimento do paciente e dos cuidadores na linha de frente das ações de prevenção, cuidado e conhecimento da doença acometida. Deste modo, o recurso deve ser adequado e adaptado de acordo com o contexto e a capacidade para sua utilização real, compreendendo o usuário e a comunidade. $\mathrm{O}$ acesso às TICs está se tornando fluente em territórios onde não havia a aquisição destas ferramentas no passado, beneficiando a assistência em saúde quando se torna tangível ao cuidador ${ }^{6}$.

Ampliando esta perspectiva, os recursos tecnológicos modernos são variados. $\mathrm{Na}$ área da saúde, estes recursos têm sido denominados por teleassistência e telessaúde, compreendendo-se como serviços de saúde à distância e atividades de educação; rede social e colaborativa web. Nesta perspectiva, destaca-se a Rede HumanizaSUS, que possibilita espaço para pessoas se comunicarem e expressarem os déficits e as potências do Sistema Único de Saúde (SUS); o uso de jogos digitais, como o serious games, interagindo com a ludicidade, o ensino e a aprendizagem; e a inclusão digital e social ${ }^{7 ; 8 ; 9 ; 10 .}$.

Considerando-se a relevância da otimização e implementação de estratégias e recursos eficazes para a atenção e cuidado à criança e ao adolescente com diagnóstico de câncer, este estudo investiga a possibilidade dos recursos tecnológicos serem incorporados à assistência prestada em serviços oncológicos de atenção a esta população. Assim, o objetivo deste estudo consiste em identificar evidências sobre o uso de tecnologias virtuais como estratégia terapêutica na otimização do cuidado à criança e ao adolescente com câncer.

\section{Métodos}

Trata-se de uma investigação Scoping Review, revisão bibliográfica que possibilita abranger temas mais amplos e visa mapear os conceitos relevantes que subsidiam uma área de pesquisa e as principais fontes e evidências disponíveis, estruturado de forma metódica e transparente, permitindo a replicação. Foram identificadas as literaturas existentes conforme as etapas da Scoping Review propostas por Arksey e O'Malley: 1) identificação da pergunta de pesquisa; 2) identificação de estudos relevantes; 3) seleção dos estudos; 4) traçar os dados; 5) coletar, resumir e relatar os resultados; e 6) consulta com especialistas (opcional) ${ }^{11}$.

A busca de referências aconteceu em outubro de 2018 e utilizou-se as bases de dados Biblioteca Virtual em Saúde (Bireme), Portal.periódicos. CAPES, PubMed e Scientific Electronic Library Online (SciELO), averiguando os critérios de inclusão e exclusão, conforme Quadro 1. Realizou-se a pesquisa através de 
cruzamento de descritores em cada base de dados, considerando: câncer, oncologia, câncer infantojuvenil, oncologia pediátrica, criança, adolescente, terapia ocupacional, telemedicina, telessaúde, saúde digital, tecnologia da informação e comunicação, e-Saúde, e-Health, realidade virtual, serious games, jogos virtuais. Respectivamente, os dados foram analisados por meio da leitura do título e resumo dos estudos encontrados conforme critérios de inclusão e exclusão. Houve eliminação de trabalhos científicos em duplicatas. A seguir, realizou-se leitura dos materiais na íntegra para elegibilidade dos resultados, sendo incluídos para revisão de escopo sete artigos.

O fluxograma representado pela Figura 1 (nos anexos) apresenta a dinâmica de busca e seleção dos estudos, conforme The Joanna Briggs Institute. Criou-se uma planilha no Excel para organização dos dados em detrimento da recomendação de The Joanna Briggs Institute, viabilizando o mapeamento dos dados, considerando: autores, ano de publicação, país/origem, objetivo/finalidade, população do estudo e tamanho da amostra, metodologia, resultados e detalhes e principais descobertas relacionadas à revisão de escopo $^{12}$.

\section{Resultados}

O Quadro 2 (nos anexos) apresenta as características gerais dos artigos encontrados. A partir do mapeamento dos dados dos sete artigos selecionados, desenvolveu-se categorias temáticas com o identitário Tecnologias da Informação, descritas a seguir.

\section{Sistema de informação em saúde}

Indexação coesa aos equipamentos e procedimentos necessários para coleta e processamento de informações, viabilizando a execução e o gerenciamento dos serviços de saúde.

O estudo de Lopes e Shmeil utiliza um software de interoperabilidade da informação no auxílio à tomada de decisão em saúde, denominada Sistema de Apoio à Decisão Clínica - Cuidado em Oncologia e Saúde com Quimioterápicos (SADCCOSQ). Trata-se de um estudo comparativo entre o software e o método convencional de orientação de cuidado com dois grupos heterogêneos e o uso do teste não paramétrico de Mann-Whitney. Neste estudo os pesquisadores analisaram que as orientações geradas aos dois grupos teve maior média de concordância naquele auxiliado pelo SADC-COSQ, condizente à estrutura das informações de cuidado, compreensibilidade e adesão às orientações ${ }^{13}$.

O estudo de Nitzlnader, Nieto, Ribelles, Brunmair, Ladenstein e Schreier se propõe a uma análise detalhada da estrutura regulamentar e das estratégias propostas pela Comissão Europeia ao dispor sobre a possibilidade de europeus irem além das fronteiras convencionais na 
busca da assistência em saúde. Ações estas que têm por objetivo a prestação de serviços, tratamento e cuidado em especialidades médicas que estão escassas, como a área de oncologia pediátrica $^{14}$.

A sistematização destas ações, segundo previsto neste acordo europeu, deve ser organizada pelos membros de estado da União Europeia. Como exemplo destas ações destacam-se 0 desenvolvimento das Redes Europeias de Referência e a criação do projeto European Expert Paediatric Oncology Reference Network for Diagnostics and Treatment (ExPO-r-NET). Os pesquisadores deste estudo investigaram a relevância da tecnologia da informação nas Redes Europeias de Referência e assistência em saúde transfronteiriça por meio da revisão bibliográfica e realizaram, também, entrevista aberta entre especialistas do projeto ExPO-r-NET, destacando as estratégias utilizadas ${ }^{14}$.

Em relação aos resultados encontrados na pesquisa bibliográfica sucedida pelos autores supracitados, destacam-se as abordagens de solução para o uso secundário de dados de assistência em saúde para pesquisa, denominada ABCD-4-E (Advanced Biomedical Collaboration Domain for Europe) e o intercâmbio de imagens clínicas, referenciada por Cross Community Access (XCA). Os requisitos encontrados na Comissão Europeia e estratégias necessárias para o projeto
ExPO-r-NET são Survivorship Passport, estratégia pautada na diretriz da União Europeia 2011/24 no artigo $12^{\circ}$, que dispõe sobre cuidados de saúde transfronteiriça; Virtual Tumour Board (vTB), ferramenta a ser desenvolvida pelo projeto ExPO-r-Net, com base na interoperabilidade; Data Provision to Clinical Research, com o objetivo de fornecer dados da assistência em saúde para pesquisa clínica; e Multimodal communication, enfocando potencializar ambiente multicêntrico ${ }^{14}$.

Considerando a arquitetura de solução das Redes Europeias de Referência, cada país possui pontos de contato nacional (NCPeH) para promover conexão e comunicação transfronteiriça, interconectados via epSOS para maior troca de informações de pacientes e tradução pelo serviço de terminologia. No $\mathrm{NCPeH}$ o acesso ao conselho virtual de tumores (vTB) acontece pelos conectores XCA/XDS-I por meio do aplicativo baseado na web (vTBApp) e por serviços de gerenciamento associados ao vTB (vTBServices). Os documentos gerados são arquivados em repositórios conectados ao $\mathrm{NCPeH}^{14}$.

Aos domínios da pesquisa clínica, usa-se uma plataforma secundária do European Network for Cancer Research in Children and Adolescents (ENCCA) que está conectada aos $\mathrm{NCPeH}$, ocorrendo transferência de documentos ao ABCD-4E. Neste meio, a identificação do paciente (eID) é modificada por pseudônimo pelo 
sistema EUPID, sendo necessário o consentimento do sujeito para o estudo clínico ${ }^{14}$.

O estudo de Silva, Souza e Couto introduz a necessidade do desenvolvimento da rede de acesso com integração aos diferentes níveis de atenção em saúde, otimizando o processo informacional de diagnóstico por câncer; a pesquisa, porém, não especifica as redes de acesso e o sistema de saúde nos seus delineamentos. As pesquisadoras realizaram análise e descrição de itinerário terapêutico de adolescentes com osteossarcoma, atribuindo os prontuários para coleta de dados, não caracterizando o formato desta ferramenta. Contudo, elas analisaram limitações quanto a ausência de dados clínicos: não há conveniência por parte dos profissionais em registrar as informações. Logo na discussão, as pesquisadoras descrevem que a apropriação do acesso para o diagnóstico de câncer infantil favorece a rede de assistência oncológica ${ }^{15}$.

O estudo de Pedrosa, Shaikh, Rivera, Ribeiro e Qaddoumi é um estudo longitudinal realizado durante quatro anos, no período de 2005 a 2009. Este estudo aborda discussão de casos de pacientes pediátricos com leucemia linfoblástica aguda (LLA), por meio da comunicação via e-mail e telefone em casos mais complexos e da produção de documentos de registros e de informações sobre o tratamento de pacientes com câncer. Foram realizadas um total de 93 reuniões por sessão de webconferência organizada pelo grupo de estudo de St. Jude Children's Research Hospital (St. Jude) e do Instituto Materno Infantil de Pernambuco (IMIP), ambas instituições parceiras desde 1993. Os dados foram categorizados em revisões de características clínicas, classificação provisória de riscos dos pacientes, complicações de tratamento, orientação de tratamento, morbidade e mortalidade e garantia de qualidade ${ }^{16}$.

Realizou-se um estudo comparativo entre dois períodos, "pré-telemedicina" (1993-2005) e "era da telemedicina" (20052009). Os resultados obtidos constataram redução da morte geral, de $31 \%$ para $12,8 \%$; de morte prematura, de $8,1 \%$ para 3,6\%; taxa de recaída, de 10,2\% para $7,9 \%$; e sobrevida geral com valores significativos, de $77 \%$ para $100 \%$. A pesquisa também permitiu a identificação de dois registros de medida de garantia de qualidade: o primeiro otimizando o padrão de atendimento de LLA no IMIP através da teleconferência; e o segundo relacionado à possibilidade de detecção de falha no tratamento após inserção do protocolo REALL-05, uma vez que nos encaminhamentos para o IMIP os pacientes se encontravam em doença avançada. Contudo, este processo em conjunto com a telemedicina resultou gradualmente na diminuição de discussões e intercorrências recorrentes sobre 0 atendimento do paciente, aperfeiçoando a aptidão clínica e 
fortalecimento das relações entre os profissionais das duas diferentes instituições $^{16}$.

O estudo colaborativo de Fortier, Chung, Martinez, Gago-Masague e Sender demandou 0 uso de tablet para o monitoramento e análise de informações de usuários em tratamento na oncologia pediátrica. O dispositivo móvel dispunha o aplicativo Pain Buddy, que possui suporte para serious games e mecanismos para coleta de informações, conectado a um servidor remoto para armazenamento dos dados, disponibilização dos dados em um portal da web. A produção das informações permite a organização de algoritmos, sincronizando, monitorando e analisando os dados ${ }^{17}$.

Ao iniciar a utilização do aplicativo Pain Buddy, o usuário fornece informações demográficas, histórico médico e de tratamento no aplicativo, navega pelas ferramentas através de um avatar tridimensional e ganha moedas virtuais que são depositadas na loja Pain Buddy como motivação e para personificar o $\operatorname{avatar}^{17}$.

A dinâmica do aplicativo constitui-se como uma ferramenta diária de sintomas, elaborada e subsidiada por instrumentos validados para avaliação da dor, como Memorial Symptom Assessment Scale (MSAS), Adolescent Pediatric Pain Tool (APPT) e questionário de intervenções de enfrentamento. As ferramentas assim produzindo são acessadas mediante critérios operacionais de interesse do usuário. O MSAS é um instrumento de porta de entrada disponível aos usuários do serviço e tem duas versões para diferentes grupos etários, de 7 a 12 anos (MSAS 7-12) e de 10 a 18 anos (MSAS 1018). Caso endosse sintomas de dor, haverá o direcionamento ao APPT para avaliação e indicação do comprometimento funcional em relação as suas atividades cotidianas. Logo, será encaminhado ao questionário de intervenções de enfrentamento para avaliar as estratégias requeridas para amenização da dor e outros sintomas ${ }^{17}$.

Em casos de identificação de ocorrências graves, os algoritmos de alerta de sintomas notificam os profissionais de saúde por e-mail. O portal da web é outra ferramenta viável aos profissionais da saúde, podendo acessar os dados em tempo real e entrar em contato com o usuário e seu responsável com recomendações para o manejo da dor de outros sintomas. O servidor notifica também o usuário do Pain Buddy quando atinge o limiar dos sintomas e que foi acionado o profissional. $O$ aplicativo pode ser usado no modo offline, mas os dados serão armazenados e criptografados, sendo enviados ao servidor quando conectado na internet ${ }^{17}$.

Com a prerrogativa de ensinar estratégias não farmacológicas, o Pain Buddy possui ferramenta de treinamento de habilidades cognitivas e comportamentais com tutoriais manejado pelo avatar personalizado do usuário, 
envolvendo imagem guiada, relaxamento muscular progressivo, respiração diafragmática, atenção plena e distração ${ }^{17}$.

Em relação a avaliação do conteúdo e o uso do aplicativo, o estudo piloto indicou satisfação dos usuários e eficácia em relação aos objetivos. Os usuários apontaram que o avatar tem design atraente, referiram explorar a loja Pain Buddy e a viabilidade do treinamento de habilidades, destacando as técnicas de respiração e as técnicas de distração como as preferidas ${ }^{17}$.

\section{Desenvolvimento de recurso tecnológico virtual}

Categoria oriunda

ao desenvolvimento das capacidades tecnológicas, incluindo métodos, técnicas e instrumentação.

O estudo de Fortier, Chung, Martinez, Gago-Masague e Sender descreve o desenvolvimento do aplicativo Pain Buddy através da abordagem colaborativa por uma equipe interdisciplinar com experiência em câncer pediátrico, controle da dor, tecnologia da informação em saúde e desenvolvimento de intervenções. Houve também a participação dos próprios pacientes com diagnóstico de câncer. O Pain Buddy foi caracterizado como serious games e utilizou-se um conjunto de ferramentas para sua criação, destacando a tecnologia de implementação Unity 3D. Como servidor usaram o Servidor de Rack PowerEdge R220, Intel® Xeon® E3-1220 v3 3,1 GHz com 8 Gb de RAM e dual HDD
500 GB Raid 1, com sistema operacional Windows Server 2012. Para o gerenciamento dos bancos de dados atribuíram o servidor versão MySQL 5.6. O portal web criado foi implementado empregando o HTML 5.0 e o PHP versão 4.5 e JavaScript para esquematizar os dados do usuário em gráficos. Para haver sincronização e monitoramento dos dados, utilizou-se o programa MySQL e PHP para organização dos algoritmos ${ }^{17}$.

O estudo de Winterling, Wiklander, Obol, Lampic, Eriksson, Pelters et al parte do desenvolvimento de uma intervenção baseada na web com o tema sexualidade e fertilidade, com participantes sobreviventes do câncer infantil e outros recrutados por indicação da equipe de enfermagem. Inicialmente houve reunião presencial entre pesquisadores e os integrantes do projeto, com apresentação e compartilhamento de histórias do câncer. O segundo encontro demandou exposição do modelo estrutural do portal Fex-Can com discussões colaborativas. Os pesquisadores enviavam informações prévias aos participantes para serem lidas e acompanhadas antes das reuniões agendadas.

Os processos resultaram na descrição das percepções dos participantes na qualidade do conteúdo, na qualidade do sistema, na qualidade do serviço e, de modo geral, no projeto de pesquisa. Abrangendo os dados, a construção do portal Fex-Can envolveu, por parte dos participantes, expressão das 
experiências e vivências com o câncer, destacando estratégias para otimizar o conteúdo informacional, baseada em evidências. Houve inclusão de atividades de cuidado, atenção à linguagem acessível e compreensível, possibilidade do usuário compartilhar suas experiências na rede, desenvolvimento de estratégias de persuasão e de dinâmica social através de fóruns, e a não normatização de informações quanto a sexualidade, (in)fertilidade, diversidade sexual, etnia, estado civil e constituição familiar. Discutiu-se o layout do portal Fex-Can e a inclusão de um profissional web designer para organização e redefinição do portal e ajustes técnicos. Os pesquisadores se responsabilizaram a averiguar publicações dos futuros usuários e a contatarem especialistas externos quando necessário. Acrescentaram no portal o e-service, correio eletrônico para dúvidas e com respostas instantâneas. $\mathrm{O}$ projeto possibilitou potência de vida aos participantes e renomearam o seu nome Life Interrupted, elaborando Fex-Can Fertility and sexuality following câncer; não enfocaram apenas nas dificuldades sexuais e de fertilidade, ampliando as informações, e salientaram a atenção aos adolescentes de 16 e 17 anos de idade e seus conflitos ${ }^{18}$.

\section{Processamento e análise digital de imagens}

O processamento de imagem com auxílio computacional viabiliza explanar e analisar o material bidimensional, técnica explorada pelo observador para sua efetividade.

O estudo de Arunachalam, Mishra, Armaselu, Daescu e Martinez apresenta abordagem para análise de segmentos de imagens na identificação de tumores viáveis e não viáveis de osteossarcoma, utilizando combinações de técnicas como segmentação de cor, Otsu thresholding e Flood-fill. A primeira etapa do processo consistiu em localizar as regiões tumorais e não tumorais e posteriormente distinguiu-se tumor viável e não viável nas regiões tumorais. A investigação das localizações se dá pela variação na distribuição de cores e na sua forma, também podendo realizar análise adicional com dados de cluster computadorizados ${ }^{19}$.

Para análise digital houve subsídio da ferramenta Aperio Scanscope, o qual possibilitou ampliar a imagem até 40X e com armazenamento SVS, variando de tamanho de $150 \mathrm{MB}$ até $1 \mathrm{~GB}$, com 109 pixels. Os pesquisadores tiveram dificuldade em encontrar um método e recurso adequado para atender ao objetivo e assim desenvolveram uma abordagem baseada em algoritmos ${ }^{19}$.

Com a aplicação das técnicas nos segmentos, os pesquisadores definiram as regiões tumorais conforme característica e quantificou a partir da abordagem, com agrupamentos de elementos semelhantes, com as seguintes classificações ${ }^{19}$ :

- $\quad \Psi 1$ - tumor viável e necrose coagulativa, ambas se assemelham e 
há análise da forma e densidade para diferenciar de tumor viável e não viável;

- $\Psi 2$ - fibrose e osteóide, classificadas como tumor não viável.

O algoritmo usado se denomina Pipeline. No início do processo utilizam a imagem SVS não processada e posteriormente o resultado é uma imagem segmentada de cores que viabiliza a identificação de tumores viáveis e não viáveis. Os pesquisadores criaram um aplicativo usando programação OpenslideJava para processar as imagens SVS, ImageJ e Java Advanced Imaging e o C\#.NET para o processamento de segmentos Otsu e Flood-fill' ${ }^{19}$.

\section{Conclusão}

As tecnologias virtuais estão interconectadas aos processos de atenção no adoecimento por câncer infantojuvenil. Agregando as estratégias e os recursos evidenciados, suas relações se apropriam às tecnologias de informação, proporcionando assistência, cuidado e qualidade de vida à criança e ao adolescente com câncer. De modo geral, expressa-se na oncologia através de ferramentas e organizações no processamento de informação, exploração de novos métodos eficazes e desenvolvimento de recursos digitais.

O uso das tecnologias virtuais no cuidado à criança e ao adolescente com câncer tem obtido o envolvimento integral deste público, aproximando-se também dos seus acompanhantes e profissionais da saúde. As estratégias têm repercutido em micro e macro soluções, favorecendo a comunicação e discussões de forma remota, a promoção da autonomia e do bem estar biopsicossocial, o lúdico, a atenção aos sobreviventes do câncer, e o processamento de imagens viabilizando 0 diagnóstico. Entretanto, salienta-se a ampliação do conhecimento das redes de acesso oncológico onde o recurso é desconhecido, objetivando registros e integração das práticas de saúde.

O mapeamento dos dados explanou os avanços e inovações aos cuidados oncológicos à população infantojuvenil a partir dos recursos de tecnologia virtual e das TICs, beneficiando o desenvolvimento de planos de ação em prol do cuidado humanizado e integral à criança e ao adolescente e favorecendo perspectivas de evolução à ciência.

\section{Agradecimentos}

Apoio financeiro: Empresa Brasileira de Serviços Hospitalares/Edital Institucional de Pesquisa da Gerência de Ensino e Pesquisa do Hospital de Clínicas da Universidade Federal do Triângulo Mineiro $N^{\circ} 01 / 2018$.

\section{Referências}

1. Pereira DS, Sousa R, Santos AC, Bezerra AMS, Gomes FLC, Santos ZMSA. A tecnologia como ferramenta promotora da saúde. In: Santos ZMSA, Frota MA, Martins ABT. Tecnologias em saúde: da abordagem teórica a 
construção e aplicação no cenário do cuidado [livro na Internet]. Fortaleza:

EdUECE; 2016 [acesso em 2019 jun 3]. p. 64-82. Disponível em:

http://www.uece.br/eduece/dmdocuments/ Ebook\%20-\%20Tecnologia\%20em\%20Sa ude\%20-\%20EBOOK.pdf\#page =12

2. Santos WA, Lourenço MLPC, Silva CD, Frizzo HCF. Terapia ocupacional em oncologia pediátrica e cuidados paliativos. In: De Carlo MMRP, Kudo AM. Terapia ocupacional em contextos hospitalares e cuidados paliativos. São Paulo: Payá; 2018. p. 145-158.

3. Kudo AM, Barros PBM, Joaquim RHVT. Terapia ocupacional em enfermaria pediátrica. In: De Carlo MMRP, Kudo AM. Terapia ocupacional em contextos hospitalares e cuidados paliativos. São Paulo: Payá; 2018. p. 127-143.

4. Instituto Nacional de Câncer José Alencar Gomes da Silva. Incidência, mortalidade e morbidade hospitalar por câncer em crianças, adolescentes e adultos jovens no Brasil: informações dos registros de câncer e do sistema de mortalidade [livro na Internet]. Rio de Janeiro: Inca; 2016 [acesso em 2017 out 20]. Disponível em:

http://www1 inca.gov.br/wcm/incidencia/20 17/pdf/versao-completa.pdf

5. Instituto Nacional de Câncer. ABC do câncer: abordagens básicas para o controle do câncer [livro na Internet]. 3o ed. Rio de Janeiro: Inca; 2017 [acesso em 2018 mai 27]. Disponível em:

http://www1.inca.gov.br/inca/Arquivos/livro -abc-3ed-8a-prova.pdf

6. Abbott PA, Barbosa SFF. Usando tecnologia da informação e mobilização social para combater doenças. Acta Paul Enferm [periódico na Internet]. 2015 [acesso em 2018 jul 10]. 28(1): [aproximadamente 3 p.]. Disponível em: http://www.scielo.br/pdf/ape/v28n1/19820194-ape-028-001-0001.pdf

7. Wen CL. Telemedicina e telessaúde: oportunidade de novos serviços e da melhoria da logística em saúde. Rev Pan Hosp [periódico na internet]. 2015 [acesso em 2019 jul 23]: p. 24-26. Disponível em: https://telemedicina.fm.usp.br/portal/wp-
content/uploads/2015/03/03132015_Revis ta_Panorama_Hospitalar_Fev_2015_pag2 4a26.pdf

8. Teixeira RR, Ferigato S, Lopes DM, Matielo DC, Sardenberg ML, Silva P et al. Apoio em rede: a Rede Humaniza SUS conectando possibilidades no ciberespaço. Interf [periódico na Internet]. 2016 [acesso em 2018 mai 30]; 20(57): [aproximadamente 12 p.]. Disponível em: http://www.scielo.br/scielo.php?script=sci_ arttext\&pid=S1414

$32832016000200337 \&$ Ing=pt\&tlng=pt

9. Neves I, Alves L, Gonzales C. Jogos digitais na classes hospitalares: desbravando novas interfaces. Obr Dig Rev Comum [periódico na Internet]. 2016 [acesso em 2017 out 25]; (10): [aproximadamente 18 p.]. Disponível em: http://www.raco.cat/index.php/ObraDigital/ article/view/315483/405589

10. Santos JMO, Santos DA, Gomes ASM. Brincando e aprendendo: uma proposta lúdica de inclusão social e digital para pacientes em serviços de oncologia pediátrica. Congresso Brasileiro de Informática na Educação; Uberlândia. Uberlândia: Anais do WIE, 2016 [acesso em 2018 jun 10]. Disponível em: http://brie.org/pub/index.php/wie/article/view/6635/ 4546

11. Arksey H, O'Malley L. Scoping studies: towards a methodological framework. Intern Jour Soci Resea Method [periódico na Internet]. 2005 [acesso em: 2019 jul 23]; 8:

[aproximadamente 14 p.]. Disponível em: https://core.ac.uk/download/pdf/56237.pdf

12. The Joanna Briggs Institute. The Joanna Briggs Institute Reviewers' Manual 2015 [livro na Internet]. Adelaide: The Joanna Briggs Institute; 2015 [acesso em 2019 jul 23]. Disponível em: https://nursing.Isuhsc.edu/JBI/docs/Revie wersManuals/Scoping-.pdf

13. Lopes VJ, Shmeil MAH. Avaliação de orientações geradas por sistema computacional a acompanhantes de pacientes pediátricos submetidos à quimioterapia. Rev Gaúcha Enferm [periódico na Internet]. 2016 [acesso em 2018 out 13]; 37(esp): [aproximadamente 
9 p.]. Disponível em:

http://www.scielo.br/pdf/rgenf/v37nspe/01

02-6933-rgenf-1983-

14472016esp67407.pdf

14. Nitzlnader $M$, Nieto $A C$, Ribelles AJ, Brunmair B, Ladenstein R, Schreier G. Interoperability architecture for a paediatric oncology European Reference Network. IOS Press [periódico na Internet]. 2016 [acesso em 2018 out 14]; 223: [ aproximadamente 7 p.]. Disponível em:

http://ebooks.iospress.nl/publication/4277 3

15. Silva TMR, Souza SR, Couto LL. Itinerário terapêutico de adolescentes com osteossarcoma: implicações para o diagnóstico precoce. Rev Min Enferm [periódico na Internet]. 2017 [acesso em 2018 out 14]; 21: [aproximadamente 6 p.]. Disponível em:

http://www.reme.org.br/exportarpdf/1164/e1028.pdf

16. Pedrosa F, Shaikh F, Rivera G, Ribeiro R, Qaddoumi I. The impact of prospective telemedicine implementation in the management of childhood acute lymphoblastic leukemia in Recife, Brazil. Telemed e-Health [periódico na Internet]. 2017 [acesso em 2018 out 8]; 23(10): [aproximadamente 7 p.]. Disponível em: https://www.ncbi.nlm.nih.gov/pmc/articles/ PMC5651971/pdf/tmj.2016.0273.pdf

17. Fortier MA, Chung WW, Martinez A, Gago-Masague S, Sender L. Pain buddy: a novel use of $m$-health in the management of children's cancer pain. Comput Biol Med [periódico na Internet]. 2016 [acesso em 2018 out 8]; 76: [aproximadamente 29 p.]. Disponível em: https://www.ncbi.nlm.nih.gov/pmc/articles/ PMC5639256/pdf/nihms909027.pdf

18. Winterling J, Wiklander M, Obol CM, Lampic C, Eriksson LE, Pelters B et al. Development of a self-help web-based intervention targeting young cancer patients with sexual problems and fertility distress in collaboration with patient research partners. JMIR Res Protoc [periódico na Internet]. 2016 [acesso em 2018 out 8]; 5: [aproximadamente 12 p.]. Disponível em: https://asset.jmir.pub/assets/5c3f4082db7 b3395ebf1ce23afd91c27.pdf

19. Arunachalam HB, Mishra R, Armaselu B, Daescu O, Martinez M. Computer aided image segmentation and classification for viable and non-viable tumor identification in osteosarcoma. Pac Symp Biocomp 2017 [periódico na Internet]. 2017 [acesso em 2018 out 08]: p. 195-206. Disponível em:

https://www.worldscientific.com/doi/pdf/10. 1142/9789813207813_0020?download=tr ue. 


\section{Anexos}

\section{Lista de figuras}

Figura 1: Fluxograma do processo de identificação e seleção de estudos relevantes.

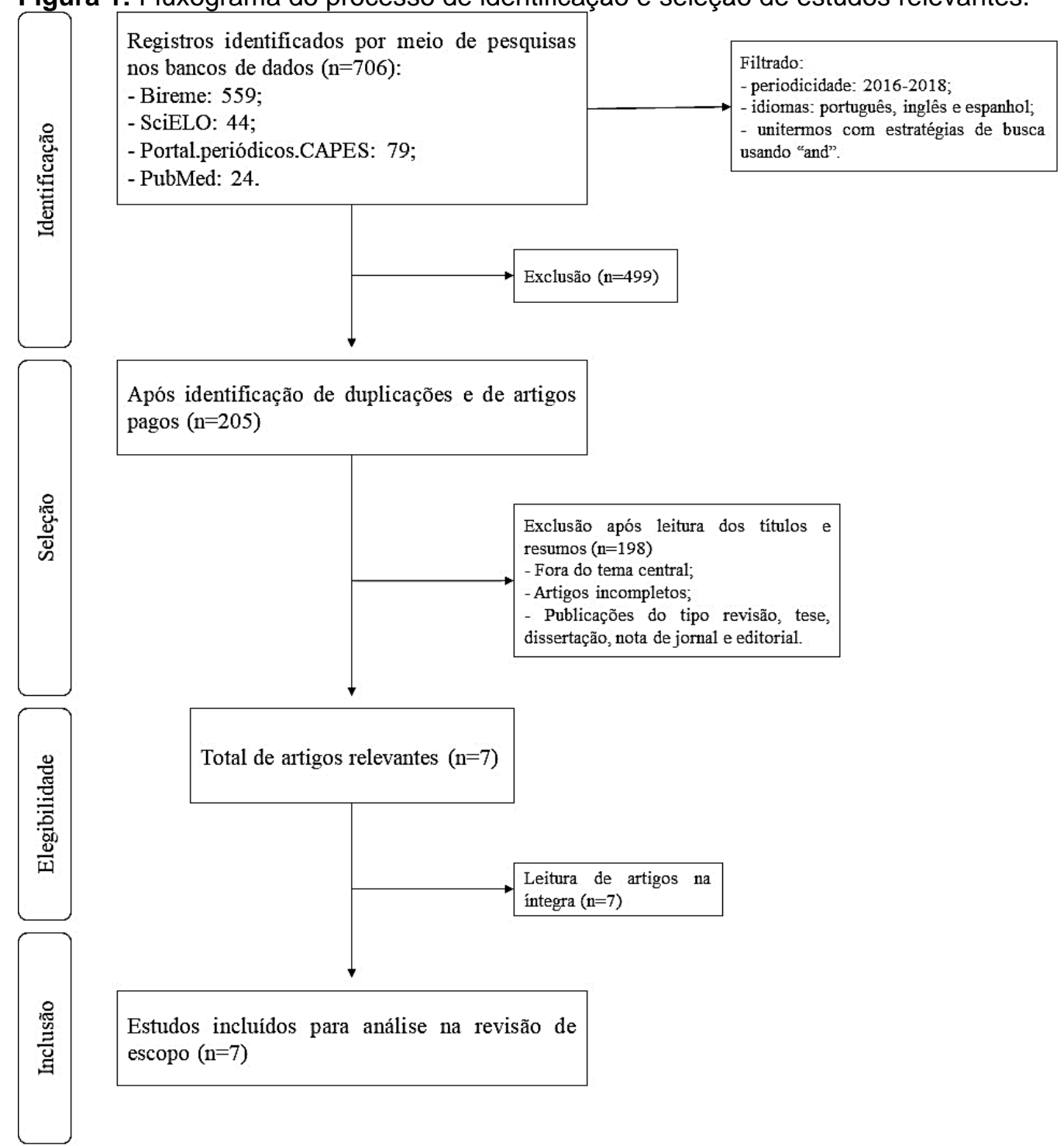

Fonte: Elaborado pelos autores. 


\section{Lista de quadros}

Quadro 1: Critérios de inclusão e exclusão.

\begin{tabular}{|l|l|}
\hline Critérios de inclusão & - Periodicidade de 2016 a 2018; \\
& - Idiomas: português, inglês e espanhol; \\
& - Artigos gratuitos; \\
& - Artigos publicados em revistas científicas presentes nas bases de dados: \\
& Bireme, SciELO, Portal.periódicos.CAPES e PubMed; \\
& - Artigos publicados em revistas científicas presentes nas bases de dados: \\
& Bireme, SciELO, Portal.periódicos.CAPES e PubMed; \\
& - Artigos originais; \\
& - Estudos nacionais e internacionais; \\
& - Estudos que abordam o uso de tecnologias virtuais no processo \\
& oncológico à criança e ao adolescente com câncer. \\
\hline Critérios de exclusão & - Duplicatas; \\
& - Artigos privados; \\
& - Artigos publicados em outros idiomas; \\
& - Estudos fora da temática central e do público; \\
& - Publicação do tipo revisão, tese, dissertação, nota de jornal e editorial. \\
\hline
\end{tabular}

Fonte: Elaborado pelos autores.

Quadro 2: Características gerais dos artigos $(n=7)$.

\begin{tabular}{|c|c|c|c|c|c|}
\hline Autores & $\begin{array}{c}\text { Ano de } \\
\text { publicação }\end{array}$ & $\begin{array}{l}\text { País/ } \\
\text { Origem }\end{array}$ & $\begin{array}{l}\text { Objetivo/ } \\
\text { Finalidade }\end{array}$ & $\begin{array}{l}\text { População do } \\
\text { estudo e } \\
\text { tamanho da } \\
\text { amostra }\end{array}$ & Metodologia \\
\hline $\begin{array}{l}\text { Arunachalam, } \\
\text { Mishra, } \\
\text { Armaselu, } \\
\text { Daescu, } \\
\text { Martinez }^{19} .\end{array}$ & 2017 & $\begin{array}{l}\text { Estados } \\
\text { Unidos }\end{array}$ & $\begin{array}{lr}\text { Apresentar uma } \\
\text { abordagem para } \\
\text { segmentar imagens } \\
\text { coradas com H\&E } \\
\text { em regiões } \\
\text { tumorais viáveis e } \\
\text { não viáveis usando } \\
\text { uma combinação } \\
\text { de r técnicas } \\
\text { (segmentação de } \\
\text { cor, Otsu } \\
\text { thresholding e } \\
\text { Flood-fill). }\end{array}$ & $\begin{array}{l}\text { Amostras } \\
\text { arquivadas de } 50 \\
\text { pacientes } \\
\text { tratados no } \\
\text { Centro Médico } \\
\text { Infantil de Dallas, } \\
\text { entre } 1995 \text { e } \\
2015 .\end{array}$ & $\begin{array}{l}\text { Metodologia } \\
\text { quantitativa. } \\
\text { Análise de } \\
\text { imagens } \\
\text { histopatológicas } \\
\text { com base em } \\
\text { métricas } \\
\text { quantitativas. }\end{array}$ \\
\hline $\begin{array}{l}\text { Fortier, } \\
\text { Chung, } \\
\text { Martinez, } \\
\text { Gago- } \\
\text { Masague, } \\
\text { Sender }^{17} \text {. }\end{array}$ & 2017 & $\begin{array}{l}\text { Estados } \\
\text { Unidos }\end{array}$ & $\begin{array}{l}\text { O estudo descreve } \\
\text { o desenvolvimento } \\
\text { e a avaliação inicial } \\
\text { do aplicativo Pain } \\
\text { Buddy, destinado a } \\
\text { otimizar o manejo } \\
\text { da dor e promover a } \\
\text { melhoria ra } \\
\text { qualidade de vida } \\
\text { de crianças e } \\
\text { adolescentes em } \\
\text { tratamento } \\
\text { oncológico. }\end{array}$ & $\begin{array}{l}\text { Crianças e } \\
\text { adolescentes } \\
\text { com idade entre } \\
8 \text { e } 18 \text { anos que } \\
\text { foram } \\
\text { diagnosticadas } \\
\text { com câncer e } \\
\text { atualmente em } \\
\text { tratamento. } \\
\text { Participaram do } \\
\text { estudo, } 12 \\
\text { crianças e } \\
\text { adolescentes, } \\
\text { cada uma } \\
\text { acompanhada } \\
\text { por rou seu } \\
\text { responsável. }\end{array}$ & Estudo piloto. \\
\hline $\begin{array}{l}\text { Lopes, } \\
\text { Shmeil }{ }^{13} \text {. }\end{array}$ & 2016 & Brasil & $\begin{array}{ll}\text { Comparar } & \text { as } \\
\text { orientações } & \\
\text { geradas com } & 0\end{array}$ & $\begin{array}{l}\text { Participaram } \\
\text { deste estudo } 08 \\
\text { enfermeiros }\end{array}$ & $\begin{array}{l}\text { Estudo } \\
\text { quantitativo. } \\
\text { Pesquisa }\end{array}$ \\
\hline
\end{tabular}

RE. SAÚD. DIGI. TEC. EDU., Fortaleza, CE, v. 4, n. 1, p. 43-59, jan./ago. 2019. 


\begin{tabular}{|c|c|c|c|c|c|}
\hline & & & $\begin{array}{l}\text { auxílio do Sistema } \\
\text { de Apoio à Decisão } \\
\text { Clínica - Cuidados } \\
\text { em Oncologia e } \\
\text { Saúde com } \\
\text { Quimioterápicos } \\
\text { (SADC - COSQ) } \\
\text { com as orientações } \\
\text { não auxiliadas por } \\
\text { tecnologia, aos } \\
\text { destinadas acampanhantes de } \\
\text { acompanh } \\
\text { crianças em } \\
\text { tratamento } \\
\text { quimioterápico. }\end{array}$ & \begin{tabular}{ll}
\multicolumn{2}{l}{ colaboradores do } \\
serviço da \\
pediatria do \\
hospital \\
filantrópico de \\
Curitiba (PR) e \\
50 \\
acompanhantes \\
de pacientes \\
pediátricos \\
oncológicos em \\
tratamento \\
quimioterápico \\
ambulatorial ou \\
internado.
\end{tabular} & $\begin{array}{ll}\text { descritiva } & \mathrm{e} \\
\text { avaliativa. } & \end{array}$ \\
\hline $\begin{array}{l}\text { NitzInader, } \\
\text { Nieto, } \\
\text { Ribelles, } \\
\text { Brunmair, } \\
\text { Ladenstein, } \\
\text { Schreier }^{14} \text {. }\end{array}$ & 2016 & $\begin{array}{l}\text { União } \\
\text { Europeia }\end{array}$ & $\begin{array}{l}\text { O artigo descreve } \\
\text { os casos de uso de } \\
\text { alto nível, os } \\
\text { requisitos centrais, } \\
\text { o estado da } \\
\text { tecnologia e uma } \\
\text { arquitetura de } \\
\text { interoperabilidade } \\
\text { correspondente. }\end{array}$ & $\begin{array}{lr}\text { Não especifica } \\
\text { população } \\
\text { estudo de } \\
\text { amostra, mas } \\
\text { expõe o estudo } \\
\text { na área de } \\
\text { oncologia } \\
\text { pediátrica. }\end{array}$ & $\begin{array}{l}\text { Análise } \\
\text { detalhada dos } \\
\text { objetivos da } \\
\text { estrutura } \\
\text { regulatória da } \\
\text { Comissão } \\
\text { Europeia (CE), } \\
\text { seguida por uma } \\
\text { revisão } \\
\text { bibliográfica } \\
\text { abrangente. } \\
\text { Entrevista não } \\
\text { estruturada com } \\
\text { diferentes de } \\
\text { especialistas } \\
\text { representando a } \\
\text { comunidade } \\
\text { ExPO-r-Net. }\end{array}$ \\
\hline $\begin{array}{l}\text { Pedrosa, } \\
\text { Shaikh, } \\
\text { Rivera, } \\
\text { Ribeiro, } \\
\text { Qaddoumi }{ }^{16} .\end{array}$ & 2017 & Brasil & 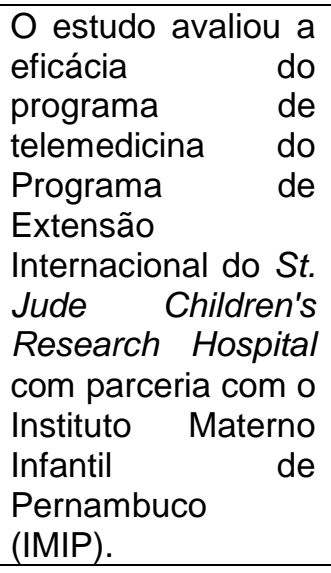 & $\begin{array}{l}\text { Crianças com } \\
\text { leucemia } \\
\text { linfoblástica } \\
\text { aguda (LLA). } \\
\text { Participaram } 163 \\
\text { pacientes } \\
\text { assistidos } \\
\text { durante o período } \\
\text { do estudo. }\end{array}$ & $\begin{array}{l}\text { Análise } \\
\text { retrospectiva } \\
\text { documental. }\end{array}$ \\
\hline $\begin{array}{l}\text { Silva, Souza, } \\
\text { Couto }^{15} \text {. }\end{array}$ & 2017 & Brasil & $\begin{array}{l}\text { Descrever } \\
\text { itinerário } \\
\text { terapêutico de três } \\
\text { adolescentes } \\
\text { portadores de } \\
\text { osteossarcoma. }\end{array}$ & $\begin{array}{l}\text { Adolescente de } \\
12 \text { a } 18 \text { anos de } \\
\text { idade com } \\
\text { diagnóstico de } \\
\text { osteossarcoma. } \\
\text { Foram utilizados } \\
\text { prontuários de } \\
\text { três adolescentes } \\
\text { atendidos no } \\
\text { ambulatório de } \\
\text { Oncopediatria. }\end{array}$ & $\begin{array}{ll}\text { Estudo } & \\
\text { descritivo, do } \\
\text { tipo relato de } \\
\text { caso. }\end{array}$ \\
\hline $\begin{array}{l}\text { Winterling, } \\
\text { Wiklander, }\end{array}$ & 2016 & Suécia & $\begin{array}{ll}\begin{array}{l}\text { Descrever } \\
\text { desenvolvimento }\end{array} & 0 \\
\end{array}$ & $\begin{array}{l}\text { Os participantes } \\
\text { foram recrutados }\end{array}$ & $\begin{array}{ll}\text { Estudo de coorte } \\
\text { nacional. } \quad \mathrm{O}\end{array}$ \\
\hline
\end{tabular}

RE. SAÚD. DIGI. TEC. EDU., Fortaleza, CE, v. 4, n. 1, p. 43-59, jan./ago. 2019. 


\begin{tabular}{|c|c|c|c|}
\hline $\begin{array}{l}\text { Obol, Lampic, } \\
\text { Eriksson, } \\
\text { Pelters et al18. }\end{array}$ & $\begin{array}{l}\text { de uma intervenção } \\
\text { baseada na Web } \\
\text { em colaboração de } \\
\text { longo prazo com } \\
\text { Parceiros de } \\
\text { Investigação de } \\
\text { Doentes (PRPs). }\end{array}$ & 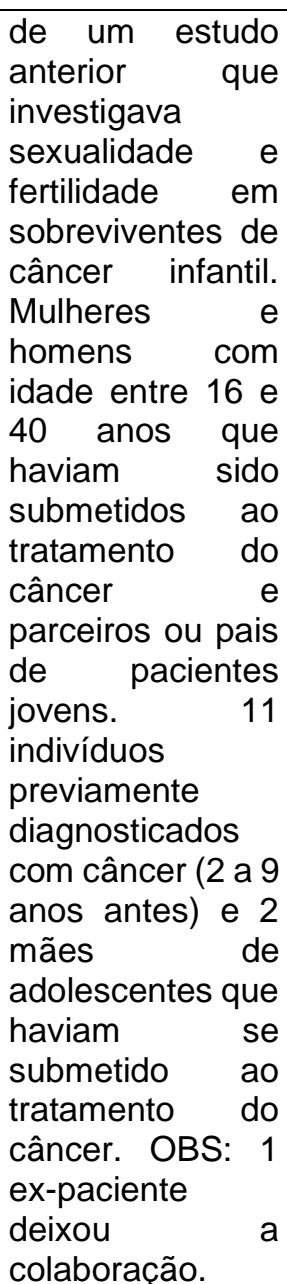 & $\begin{array}{l}\text { estudo é } \\
\text { baseado na } \\
\text { estrutura } \\
\text { holística para o } \\
\text { desenvolvimento } \\
\text { de tecnologias e- } \\
\text { Health por van } \\
\text { Gemert-Pijnen et } \\
\text { al e se concentra } \\
\text { na etapa de } \\
\text { design do } \\
\text { modelo, ou seja, } \\
\text { processo } \\
\text { participativo co- } \\
\text { criativo } \\
\text { construção de da } \\
\text { intervenção } \\
\text { baseada na } \\
\text { Web. }\end{array}$ \\
\hline
\end{tabular}

Fonte: Elaborado pelos autores.

RE. SAÚD. DIGI. TEC. EDU., Fortaleza, CE, v. 4, n. 1, p. 43-59, jan./ago. 2019. 


\section{Como citar este artigo}

Silva GHda, Frizzo HCF. O uso de tecnologias virtuais no cuidado à criança e ao adolescente com câncer: scoping review. Revista de Saúde Digital e Tecnologias Educacionais. [online], volume 4, n. 1. Editor responsável: Luiz Roberto de Oliveira. Fortaleza, mês e ano, p. 43-59. Disponível em: http://periodicos.ufc.br/resdite/index. Acesso em "dia/mês/ano".

Data de recebimento do artigo: 18/06/2019

Data de aprovação do artigo: 23/07/2019 